\title{
Modulating what is and what could have been: The effect of transcranial direct current stimulation on the evaluation of attained and unattained decision outcomes
}

\author{
Mascha van 't Wout ${ }^{1,2,3}$ (D) $\cdot$ Hannah Silverman ${ }^{3,4}$
}

Published online: 10 October 2017

(C) Psychonomic Society, Inc. 2017

\begin{abstract}
The affective evaluation of decision outcomes, whether attained (e.g., disappointment) or based on the conscious realization that a decision made differently would have led to a better or worse outcome (e.g., regret), greatly influence future decisions. Prior research has demonstrated a role of the medial and orbitofrontal cortex (M/OFC) in decision valuation and the experience of regret and relief. Here we examined whether inhibitory transcranial direct current stimulation (tDCS) could dampen the experience of decisioninduced affect, with a focus on regret and relief. Thirty-eight participants completed a previously used gambling task and were asked to rate their happiness with attained outcomes of a chosen gamble before and after being shown unattained, counterfactual outcomes (i.e., what would have happened had they selected the other gamble). The difference in happiness rating before and after revealing these unattained counterfactual outcomes was taken as a measure of regret (negative
\end{abstract}

Electronic supplementary material The online version of this article (https://doi.org/10.3758/s13415-017-0541-9) contains supplementary material, which is available to authorized users.

Mascha van 't Wout

maschavantwout@gmail.com

1 Department of Psychiatry and Human Behavior, Warren Alpert Medical School, Brown University, 345 Blackstone Blvd, Providence, RI 02906, USA

2 Center for Neurorestoration and Neurotechnology, Providence VA Medical Center, Providence, RI, USA

3 Department of Cognitive, Linguistic \& Psychological Sciences, Brown University, Providence, RI, USA

4 The Taub Institute for Research on Alzheimer's Disease and The Aging Brain, Columbia University Medical Center, New York, NY, USA shift) or relief (positive shift). During this task, 20 participants received $2 \mathrm{~mA}$ cathodal tDCS over EEG coordinate Fp1 for 20 minutes, and 18 participants received sham stimulation over the same location. Linear mixed-model results showed that, compared to sham, participants who received cathodal tDCS reported less intense emotions in response to attained as well as counterfactual outcomes. These findings were not due to the groups differing in the gambles they selected or attained monetary outcomes, demonstrating that tDCS can modulate decision-induced (counterfactual) affect. This may have implications for the ability to modulate value-based decisionmaking using brain stimulation techniques more broadly.

Keywords Decision-making · Emotion · Prefrontal cortex · Transcranial direct current stimulation

The evaluation and accompanying emotions of anticipated and experienced outcomes are crucial to flexible decisionmaking (Mellers \& McGraw, 2001). Making a deliberate decision between two or more options does, however, require rejecting the alternatives. This creates uncertainty, as we often do not know which decision will provide the outcome of highest value (Bell, 1982). Taking the example from Simonson (1992), imagine that, after some deliberation, you decide to purchase a new pair of expensive pants, only to find out 2 days later that the pants went on sale. In this example, the outcome of the alternate decision (e.g., pants on sale) became available, allowing you to reevaluate your attained outcome (e.g., expensive pants), and resulting in feelings of regret. This general ability to reason about outcomes had a different decision been made ("what might have been . ..") is termed counterfactual reasoning. Thus, regret and relief can be described as counterfactual emotions based on the conscious realization that a decision made differently would have led 
to a better or worse outcome, respectively (Gilovich \& Medvec, 1995; Zeelenberg, 1999). Regret and relief involve an internal reflection on actions for which we feel some sense of responsibility (Connolly, Ordonez, \& Coughlan, 1997; Zeelenberg, Van Dijk, \& Manstead, 1998), for example, it was you who bought the pants. Moreover, the fear of making a regretful choice influences how we decide (Bell, 1982; Zeelenberg, 1999; Zeelenberg, Van Dijk, Manstead, \& van der Pligt, 2000), and next time you might wait for a sale.

Neuroimaging and neuropsychological research demonstrate that the experiences of regret and relief activate the orbitofrontal cortex (OFC; Coricelli, Dolan, \& Sirigu, 2007). Specifically, the ventral caudate and the ventral medial or medial OFC showed greater activation when people were confronted with better alternative outcomes in a particular gamble paradigm. In fact, activation in the medial OFC correlated positively with the amount of regret or relief experienced, and over time participants started to avoid regretinducing options (Coricelli et al., 2005). Moreover, Chua, Gonzalez, Taylor, Welsh, and Liberzon (2009) reported that the increased lateral OFC activity was specific to regretinducing situations as compared to disappointment. Finally, Camille et al. (2004) investigated patients with lesions in the basal and ventromedial sector of the prefrontal cortex (including Brodmann's areas 10, 11, 32, 24, and 47) and found that these patients did not report experiencing regret, nor were they able to alter their behavior to avoid regret-inducing situations in the future. Taken together, these studies highlight the importance of the OFC for evaluating situations in which people may experience counterfactual emotions such as regret and relief, and to adapt their behavior accordingly.

The importance of the OFC for the processing of counterfactual emotions fits with the broader view associating the OFC with decision-making behavior and valuation over time. Prior studies have highlighted a central role for ventromedial and orbitofrontal areas in assigning and tracking (predictive) value to objects and outcomes in the world (Bechara, Damasio, \& Damasio, 2000; Frank \& Claus, 2006; Rolls \& Grabenhorst, 2008; Wallis, 2007) and forming associations between affectbased responses and abstract information about outcomes (Roy, Shohamy, \& Wager, 2012; Tsuchida, Doll, \& Fellows, 2010; Ursu \& Carter, 2005). If the OFC, as part of a neural network, plays a crucial role in the valuation of decision outcomes as well as reevaluation or affective updating, it can be hypothesized that modulating OFC activity will influence the experience of decision-induced disappointment, happiness, regret, and relief. In line with this hypothesis are the results reported by Huey et al. (2015), who demonstrated that, in individuals with neurodegenerative diseases, gray matter volume reductions in the right medial and lateral OFC were associated with apathy. Clinically, there are reasons why modulation of emotional responses to counterfactual outcomes (hypothetical "counterfactual emotions") might be desirable. Excessive feelings of regret (or fear thereof) have been associated with clinical depression and anxiety disorders, such as obsessive-compulsive disorder (Gillan et al., 2014; Rasmussen \& Eisen, 1992; Roese et al., 2009; Sachdev \& Malhi, 2005). Addiction, on the other hand, has been associated with undervaluation of counterfactual outcomes (i.e., side effects, and an overvaluation of immediate reward; Volkow \& Fowler, 2000).

One accessible, well-tolerated, noninvasive neuromodulatory technique is transcranial direct current stimulation (tDCS). During tDCS, a weak electrical current $(1-2 \mathrm{~mA})$ is applied to the scalp to modulate the excitability of neurons in the brain by changing membrane potentials in localized areas (Nitsche et al., 2008). While cathodal tDCS is thought to generally decrease neuronal excitability, anodal tDCS is associated with increased excitability under a targeted brain region (Nitsche \& Paulus, 2000). Recently, we have applied tDCS, aimed to target the ventromedial prefrontal cortex, to modulate extinction learning and memory of conditioned fear (van't Wout et al., 2017; van't Wout et al., 2016), highlighting the ability of tDCS to possibly reach these brain structures to modulate salience-based responses.

In this study, we examined whether the application of $2 \mathrm{~mA}$ cathodal tDCS over area Fp1 (anode over area PO8) of the 1020 EEG coordinate system while individuals were engaged in a counterfactual gambling task modulated the experience of decision-induced affect, including regret and relief. We hypothesized that the application of cathodal tDCS would reduce the reported intensity of decision-induced affect and counterfactual affect as compared to sham stimulation. However, given that our gambling task was not designed to include learning to avoid the experience of disappointment or regret, we hypothesized tDCS would not influence the actual decisions made. Hence, tDCS would specifically influence the evaluation of outcomes and counterfactual outcomes.

\section{Materials and Method}

\section{Participants}

Thirty-nine participants were recruited through advertisements posted on the Brown University campus and online through Craigslist. Potential participants were prescreened on the phone for inclusion/exclusion criteria. Exclusion criteria involved any tDCS contraindications (e.g., current, or history of, neurological disease or closed-head injury; implanted electronic hardware or metal in the cranial cavity; broken skin or other lesions in the area of the electrodes; presence of holes in the skull made by trauma or surgery; pregnancy); current, or history of, psychotic disorder; depressive disorder (major depressive disorder/bipolar disorder); and current substance abuse/dependence or use of prescribed psychotropic medications. The Brown University Institutional Review approved study procedures and materials consistent with the Declaration of Helsinki, and written 
informed consent was obtained prior to onset of any study procedures. After providing informed consent, participants were rescreened for any exclusionary factors, and subsequently randomly assigned to receive either active tDCS $(N=20)$ or sham stimulation $(N=19)$.

\section{Gambling task}

All participants engaged in 96 rounds of a gambling task programed in E-Prime. Our gambling task was based on the task used by Camille et al. (2004) and Coricelli et al. (2005) as a method to induce and measure counterfactual regret and relief. In order to increase their emotional stake in the task, participants were told they would be compensated with $\$ 10$ for the 1hour study and could receive up to an additional $\$ 5$ depending on their outcomes. However, all participants were compensated with the full $\$ 15$ for participation.

In each of the 96 rounds, participants were shown and asked to choose between two wheels of fortune that varied in probabilities of obtaining one of two outcomes. The possible outcomes were "Losing \$32," "Losing \$8," "Winning $\$ 8$," and "Winning \$32." The probabilities were either .5 and .5 or .2 and .8 (see Supplementary Materials for the full list of trials in the presented order; trial order was fixed). After participants made a choice by selecting one wheel of fortune by button press, participants saw a spinner go around that landed on one outcome. This was the attained outcome. Participants were asked to rate their happiness with this attained outcome on a scale from -50 (very unhappy) to +50 (very happy), with zero being neutral, that could be entered with the keyboard. After submitting their rating, a spinner appeared in the wheel of fortune they did not choose, and participants were shown the unattained "counterfactual" outcome had they chosen this other wheel of fortune. Participants were then asked to again rate their happiness with their original attained outcome (see Fig. 1a).
Rounds where the counterfactual outcome was greater than the attained outcome were intended to induce regret, whereas rounds where the attained outcome was greater than the counterfactual outcome were intended to induce relief. Participants' experiences of regret and relief were quantified by taking the difference between happiness ratings in response to the attained outcome before and after the counterfactual outcome was revealed to obtain a happiness-difference rating. In other words, the happiness-difference rating was calculated by subtracting the happiness rating in response to the attained outcome of a chosen gamble before revealing the unattained, counterfactual outcome from the happiness rating in response to the attained outcome after we revealed the unattained, counterfactual outcome on every trial. This resulted in a happiness-difference rating in which negative values indicated feelings of regret and positive values indicated relief. At the end of the task, participants were debriefed with a description of the goals of the study and an explanation that the outcomes were fixed, and therefore the task was not an accurate representation of their skill.

\section{tDCS}

tDCS was delivered using a built-in rechargeable battery-driven NeuroConn DC-Stimulator Plus (NeuroConn Inc, Ilmenau, Germany). We used a 1 (cathode) $\times 1$ (anode) unilateral electrode setup (Nasseri, Nitsche, \& Ekhtiari, 2015) with each electrode placed in a $5 \times 5 \mathrm{~cm}\left(25 \mathrm{~cm}^{2}\right)$ reusable sponge pocket saturated with $0.9 \%$ normal saline. Sponges with electrodes were attached to the participant's skull using a rubber headband.

The cathodal electrode was placed over Fp1 of the 10-20 EEG electrode coordination system, and the anodal electrode was placed over PO8. This montage was chosen to deliver current to the OFC based on modeling results from tDCSExplore Soterix Medical neurotargeting software (Kempe, Huang, \& Parra, 2014; see Fig. 1b), while preventing anodal stimulation over the prefrontal cortex (see also Abend et al., 2016; Civai, a

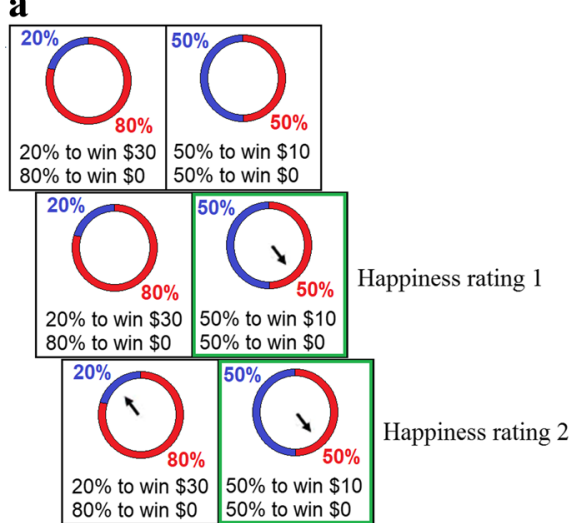

Fig. 1 Example of one trial in the gambling task (a) and current density modeling results of cathodal electrode over EEG coordinate Fp1 and anodal electrode over EEG coordinate PO8, in MNI coordinate taken

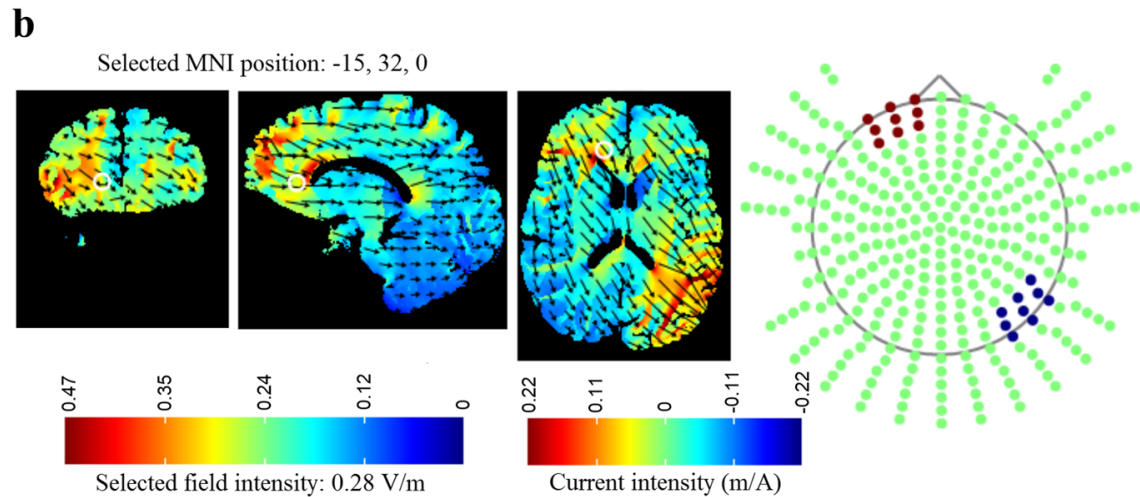

from Coricelli et al. (2005), generated by tDCS Explore from Soterix Medical (b). (Color figure online) 
Miniussi, \& Rumiati, 2015; Zheng et al., 2016, for similar montages that aim to avoid prefrontal stimulation with the opposing electrode). A close to similar electrode montage was successfully used to augment extinction of conditioned fear previously (van’t Wout et al., 2017; van't Wout et al., 2016), suggesting that this type of montage might be able to modulate medial prefrontal cortex (mPFC)/OFC-based processes.

To prevent side effects, the skin under the stimulation sites was lightly cleaned with alcohol and inspected for lesions or abnormalities. Participants were instructed to notify the experimenter of any discomfort and informed that stimulation would be discontinued if discomfort occurred. To ensure tDCS tolerability to study procedures, all participants initially received brief stimulation ( $1 \mathrm{~mA}$ for 30 seconds, with a ramp up/down over 15 seconds each).

Participants were randomized to receive either active tDCS or sham stimulation during the gambling task. Active tDCS consisted of 20 minutes of 2-mA intensity (current density 0.8 $\mathrm{A} / \mathrm{m}^{2}$ ) started simultaneously with gambling task onset. This stimulation duration was chosen to stay well within the known safety limits of tDCS as used previously (Brunoni et al., 2011; Bikson, Datta, \& Elwassif, 2009), but had the disadvantage that gambling task duration outlasted tDCS duration for the majority of participants. Although prior research suggests that tDCS results in aftereffects that last at least as long as the actual stimulation duration and probably longer (Nitsche et al., 2003; Nitsche \& Paulus, 2001), the trial on which tDCS finished during the gambling task was recorded for analyses (see Supplementary Materials). Sham stimulation consisted of 1-mA intensity for 30 seconds, also started simultaneously with gambling task onset. tDCS device ramp up/ down to/from the respective maximum intensity was $15 \mathrm{sec}-$ onds each in both the active tDCS and sham condition.

\section{Power assessment}

In order to assess prospective power, we drew upon literature on tDCS, decision-making, and decision-induced affect. Studies that examined tDCS-modulated decision-making targeting the dorsolateral prefrontal cortex show significant effects in samples ranging from 9 to 12 participants each (Boggio et al., 2010; Fecteau, Knoch, et al., 2007; Fecteau, Pascual-Leone, et al. 2007; Hecht, Walsh, \& Lavidor, 2010). More recent studies, also targeting the dIPFC examining the effects of tDCS on decision-making, report significant findings in samples of 16 to 20 participants (Cheng \& Lee, 2016; Ye et al., 2016). Finally, relevant for our design, a tDCS study aiming to target the mPFC observed significant tDCS modulation on value-based decision-making in 16 participants (Hämmerer, Bonaiuto, Klein-Flügge, Bikson, \& Bestmann, 2016). With respect to the other field of interest, regret, Camille et al. (2004) observed that subjective experience of emotions in the gambling task are significantly affected by values of the attained as well as the unattained outcomes in a healthy control sample of 18 participants. Moreover, Coricelli et al. (2005), who focused mainly on neuroimaging data, evaluated emotional reactions to one presentation of each pair of attained-unattained outcomes in the 15 participants who were subsequently scanned during the task. This observation suggests that the number of trials (96) we employed should be sufficient to observe feelings of disappointment, regret, and relief in our task. Taken together, we determined that a sample size of at least 18 participants in either tDCS group would provide sufficient power.

\section{Data quality}

During data quality checks, before statistical analysis, data from one participant in the sham group demonstrated apparent misunderstanding of the task. Specifically, data quality inspection revealed that this individual did not provide a secondary happiness rating after the counterfactual outcome was displayed on 16 out of the total 96 trials, resulting in no data on $16.7 \%$ of trials. On the remainder of the 80 trials where, after reveal of the counterfactual, a happiness rating was entered, this individual appeared to have consistently rated happiness with the counterfactual outcome as if it was the outcome attained. This was based on the observation that happiness scores increased when the counterfactual outcome was greater (e.g., won \$32) than the attained outcome (e.g., lost $\$ 8$ ) and decreased when the counterfactual was smaller (e.g., lost \$32), resulting in a significant negative correlation between counterfactual monetary outcomes and the happinessdifference rating, $r(78)=-0.23, p=.04$, something that was not observed in any of the other participants. For these reasons, we excluded the data from this participant from all analyses, resulting in groups of 18 participants who received sham stimulation and 20 participants who received active tDCS.

Our data quality check also revealed that on trials numbers 31,50 , and 96, the attained outcome and counterfactual outcome was identical (e.g., losing $\$ 8$; see Supplementary Materials), resulting in no induction of regret or relief on these trials. This was due to programming errors in the task, and these trials were therefore excluded from all analyses.

We further observed the presence of happiness-difference rating values of zero. Values of zero on this measure either indicated (1) no regret or relief was experienced, or (2) inability to update ratings due to limited range of our rating scale. For example, winning/losing \$32 may have resulted in a maximum/minimum initial happiness rating of $+50 /-50$ that could not further increase/decrease after the counterfactual outcome was revealed. Thus, the potential "happiness" shift after revealing the counterfactual outcome when initial happiness ratings were either +50 or -50 could not be detected. For that reason, only when analyzing the happiness-difference rating, we excluded trials on which a happiness-difference rating 
of zero was due to both happiness ratings being either +50 or -50 , because we could not determine whether a lack of change on the rating scale was due to limits of our scale or effects of tDCS in these cases. Across all participants, $30.9 \%$ of trials were excluded for this reason. There was a significant difference between groups, with fewer trials excluded for this reason in the active tDCS as compared to the sham stimulation group, Wald $\chi^{2}(1)=6.41, p=.01$.

\section{Statistical analyses}

Group differences on demographics and tDCS blinding effectiveness were tested using $t$ test, chi-square, or nonparametric Mann-Whitney $U$ tests. During the gambling task, each participant completed multiple gambling trials (maximum 96). We therefore applied a linear mixed model for analyses of the gambling task data using SPSS Version 24. Mixed models account for shared variance within subjects while modeling between-subject differences and allow examination of behavioral responses while adjusting for correlations due to repeated observations within participants. Although the independent and dependent variables changed depending on the analysis performed (as outlined below), the variable subject was always entered as a correlated random-effects variable. The influence of tDCS on the following different aspects of gambling task behavior were tested:

1. Gamble selection choice behavior and monetary outcomes. A binary logistic generalized estimating equations model was used to examine whether tDCS may have influenced participants' choices in the task. ANOVA was used to test a group difference in the amount of money attained in the task. A linear mixed model was used to examine the effect of tDCS on reaction times.

2. Initial happiness ratings. A linear mixed model was used to examine whether tDCS affected happiness ratings with wins $(\$ 8 / \$ 32)$ or losses $(-\$ 8 /-\$ 32)$, that is, before the counterfactual was revealed. Followed by post hoc tests to examine tDCS effects on wins and losses separately, and a separate analysis to test whether tDCS affected happiness rating reaction time.

3. Happiness-difference ratings. A linear mixed model was used to examine whether tDCS affected the experience of regret and relief. Followed by post hoc analyses to examine tDCS effects on regret and relief separately, and a separate analysis to test whether tDCS influenced reaction time to enter the happiness rating for the second time.

A two-sided alpha level of 0.05 was applied to determine significance in all analyses. Happiness (difference) rating plots demonstrated no outliers or data points that contributed excessive influence or leverage. Reaction times less than $250 \mathrm{~ms}(N=1)$ were excluded as well as trials with a reaction time $z$ score $>4$ (i.e. RT $\geq 10 \mathrm{~s}, N=179$ or $<5 \%$ of all trials) as these were likely outliers based on box plots and stem-andleaf plots.

\section{Results}

\section{Demographics}

Mean age for participants in the active tDCS group was 24.85 years $(S D=9.05)$ and 24.05 years $(S D=6.35)$ in the sham group, which did not differ significantly, $t(37)=0.32, p=.75$. Active and sham groups also did not differ in gender distribution (Active tDCS: 11 females, nine males; Sham: seven females, 12 males); $\chi^{2}(1)=1.29, p=.26$, or educational level (Active tDCS: all college educated; Sham: one < college, 16 college-educated, two graduate degree); Mann-Whitney $U(1)$ $=180, p=.54$.

\section{tDCS}

The NeuroConn tDCS device provides impedance levels to indicate electrode contact quality. Average impedance obtained during active tDCS for this study was $5.15 \mathrm{k} \Omega(S D=3.12)$, which is well below the $55-\mathrm{k} \Omega$ maximum allowed by the device. In order to assess tDCS tolerability, participants' reaction and skin under the electrodes after tDCS/sham was assessed, including inquiring whether they thought they received active tDCS or sham. One participant in the active tDCS group reported feeling heat under the frontal, cathodal electrode. All participants reported feeling a mild tingling/ itchy sensation during stimulation, and all participants enrolled in the active tDCS group demonstrated slight skin redness under the electrodes. No other adverse effects were reported during or after administration of tDCS.

In order to evaluate blinding effectiveness, we tested whether participants accurately guessed which treatment condition (active tDCS or sham) they were assigned to. There was no significant difference of actual tDCS group (active tDCS or sham) on guessed treatment condition, $\chi^{2}(1)=2.20, p=.14$. This suggests that participants were not able to accurately guess whether they had received active tDCS or sham.

\section{Gambling task}

\section{Effect of tDCS on task choice behavior and monetary outcomes}

First, we wanted to test whether tDCS would influence participants' choices in the task, that is, selecting the gamble with the higher expected value, which is the possible monetary outcome multiplied by likelihood of that outcome. To test this, we performed a binary logistic generalized estimating equations model 
across all trials, with subject as a repeated-subject variable, gamble chosen (whether participants selected the left or right gamble on each trial) as a dependent variable, and tDCS group and expected value (whether the left or right gamble had a higher expected value) as predictors. There was no significant main effect of tDCS group, $\chi^{2}(1)=0.56, p=.46$, a significant main effect of expected value, $\chi^{2}(1)=675.49, p<.0001$, Cohen's $d=$ 2.54 , and no significant tDCS Group $\times$ Expected Value interaction, $\chi^{2}(1)=0.59, p=.44$. This suggests that tDCS did not influence whether participants selected the right or left gamble. and groups were equally likely to choose the gamble with the higher expected value. Consistently, there was no significant difference between active tDCS and sham groups on the amount of money attained in the task, Mean active tDCS: $\$ 5.97$ ( $S D=$ 1.27); mean sham: $\$ 6.53(S D=1.06) ; F(1,36)=2.14, p=.15$, $\eta_{\mathrm{p}}^{2}=0.06$.

A separate linear mixed-model analysis, with subject as a correlated random effects variable, reaction time for gamble selection as dependent variable, and tDCS group as factor demonstrated no significant effect of tDCS on response time to select a gamble, $F(1,36.06)=1.45, p=.24$. These data suggest that tDCS did not affect gamble selection.

\section{Effect of tDCS on initial happiness}

Next, we tested whether tDCS might have influenced the initial happiness rating (Rating 1). Across all trials, we performed a linear mixed model, with subject as a correlated random effects variable, Happiness Rating 1 as a dependent variable, and tDCS group and whether the attained outcome was a win ( $\$ 8$ or $\$ 32$ ) or loss $(-\$ 32$ or $-\$ 8)$ as factors (both tDCS and attained outcome were binary variables). There was no significant main effect of tDCS group, mean active tDCS: $12.92(S D=33.21)$; mean sham: $15.66(S D=38.18) ; F(1,36.65)=0.66, p=.42$, Cohen's $d=0.07$. There was a significant main effect of attained outcome, $F(1,3357.97)=11$ 224.91, $p<.0001$, Cohen's $d=$ 3.53, as well as a significant Attained Outcome $\times$ tDCS Group interaction, $F(1,3357.97)=110.06, p<.0001$ (see Fig. 2a). This

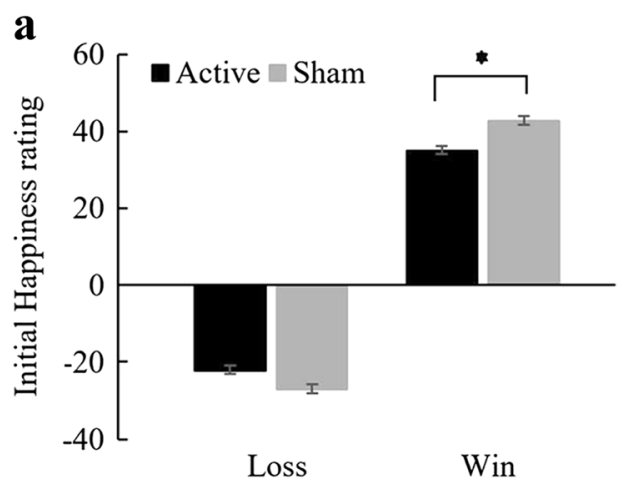

Fig. 2 a Average happiness ratings ( $y$-axis) as a function of attained outcomes being a loss or a win ( $x$-axis) separated for active tDCS and sham groups. b Average happiness-difference ratings ( $y$-axis) as a suggests that although participants were generally happier with wins than losses, which elicited feelings of disappointment, tDCS had a dampening effect on these ratings. Post hoc tests demonstrated that the tDCS-group difference was significant when selecting wins only, $F(1,35.99)=7.03, p=.01$, Cohen's $d=0.5$, but not losses, $F(1,36.02)=1.29, p=.26$, Cohen's $d=$ 0.24 .

We also tested whether tDCS could have influenced reaction times on entering an initial happiness rating. Linear mixed-model analysis, with subject as a correlated random effects variable, reaction time for happiness rating as dependent variable, and tDCS group as factor demonstrated no significant effect of tDCS, $F(1,35.98)=0.37, p=.55$. (See Supplementary Materials for model results with attained outcome as an additional factor.)

\section{Effect of tDCS on counterfactual regret and relief}

To test whether cathodal tDCS modulated the experience of counterfactual emotions, we performed a linear mixed model, with subject as a correlated random effects variable, happiness-difference rating as a dependent variable, and counterfactual outcome (regret or relief) and tDCS group as factors. Results revealed a significant main effect of counterfactual outcome, mean regret: $-9.47(S D=22.32)$, mean relief: 7.86 $(S D=22.41), F(1,2039.72)=328.77, p<.0001$, Cohen's $d=$ 0.78; a nonsignificant main effect of tDCS group, mean active tDCS: $-0.08(S D=19.93)$, mean sham: $-0.98(S D=28.99)$, $F(1,29.01)=0.18, p=.68$, Cohen's $d=0.04$; and a significant Counterfactual Outcome $\times$ tDCS Group interaction, $F(1$, 2039.72) $=9.04, p=.003$ (see Fig. 2b). This suggests that, across regret and relief trials, participants receiving active tDCS reported a reduced intensity of counterfactual emotions as compared to participants who received sham when confronted with counterfactual "what could have been" outcomes.

Post hoc linear mixed models aimed to test the effect of tDCS on regret and relief trials separately, demonstrated that

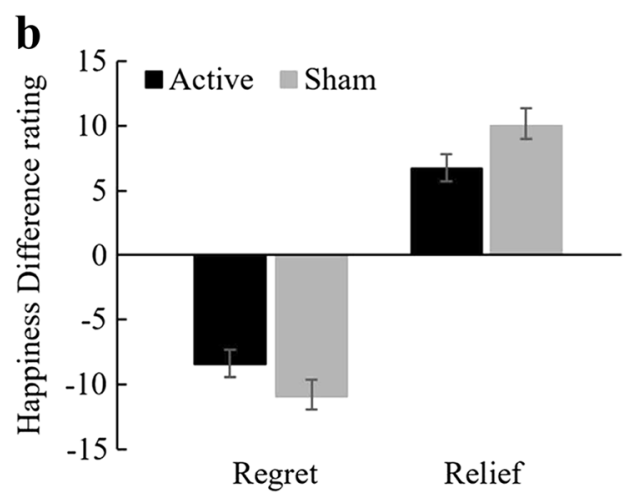

function of counterfactual outcomes resulting in regret or relief ( $x$-axis) separated for active tDCS and sham groups. Error bars represent standard error. * Indicates significant post hoc tDCS group difference 
tDCS did not significantly affect the experience of regret, $F(1$, $28.51)=0.56, p=.46$, or relief, $F(1,32.38)=0.29, p=.59$, when each was analyzed separately. This seems to suggest that the modulation of counterfactual emotions due to tDCS is modest, only reaching significance when regret and relief are taken into consideration simultaneously, as tDCS dampens both these feelings to some degree.

Finally, linear mixed-model analysis, with subject as a correlated random effects variable, reaction time for rating happiness after the counterfactual outcome was revealed as a dependent variable, and tDCS group as a factor, demonstrated no significant effect of tDCS, $F(1,35.23)=2.88, p=.10$. (See Supplementary Materials for model results with counterfactual outcome as an additional factor.)

\section{Discussion}

Our results show that individuals who received cathodal tDCS over coordinate Fp1 (anodal electrode P08) reported significantly less intense happiness ratings in reaction to attained outcomes and a smaller change in subjective happiness ratings with attained outcomes after counterfactual "what could have been" outcomes were revealed than participants receiving sham stimulation. This dampening effect of tDCS appeared to occur in either direction, and happiness or disappointment as well as regret or relief appeared to be less intense in participants who received active tDCS as compared to sham. However, post hoc tests demonstrated that the dampening effect of tDCS only reached statistical significance when rating initial happiness with attained wins. Post hoc tests examining the effect of tDCS on disappointment with attained losses, or regret and relief separately were nonsignificant, suggesting that the effect of tDCS on counterfactual emotions appeared to be modest, reaching significance when regret and relief were taken into consideration simultaneously.

The difference in initial happiness ratings or counterfactual change scores between the active tDCS and sham group was not due to a significant difference in groups making different decisions, and participants in both groups tended to select the gamble option with the higher expected value. Moreover, there were no significant differences between groups on reaction times for gamble decisions, or in the average attained outcomes. This is in line with Bogdanov, Ruff, and Schwabe (2015), who reported that anodal or cathodal tDCS targeting the dorsolateral prefrontal cortex did not influence choices made in an investment task as compared to sham. Hence, group differences in reported happiness - and to some extent disappointment, regret, and relief-were not due to participants in one group doing "better" (i.e., higher attained outcomes) than participants in the other. Taken together, these data support the conclusion that cathodal tDCS may dampen the emotional experience of decision-induced affect and counterfactual emotions specifically.

To our knowledge, these findings are the first to indicate that electrical noninvasive neuromodulation aimed to target the OFC can modulate the experience of both decisioninduced affect and counterfactual emotions. Furthermore, our data are in line with prior research indicating the vital importance of the OFC in the experience of regret and relief (Camille et al., 2004) as well as OFC/mPFC-based neurocircuitry in value-based decision-making more broadly (Fellows, 2011) and related to updating changing values over time (Fellows \& Farah, 2005), risk assessment (Clark et al., 2008), and specific social-emotional moral decisions (Moretto, Làdavas, Mattioli, \& Di Pellegrino, 2010). Extrapolating to this broader area of research on value based behaviors, our findings are consistent with our earlier research on the ability of tDCS to modulate Pavlovian value-based extinction learning (Rangel, Camerer, \& Montague, 2008) in healthy volunteers (van't Wout et al., 2016) and extinction recall in Veterans with posttraumatic stress disorder (van't Wout et al., 2017), using a close to similar tDCS montage. Along the same lines, Ly et al. (2016) recently demonstrated that tDCS influenced affective biasing of instrumental action (Ly et al., 2016). Taken together, these data are relevant for the potential of noninvasive brain stimulation techniques, and tDCS in particular, given its associated safety profile (Bikson et al., 2016), to modulate intrinsic affective and cognitive processes during treatments for neuropsychiatric disorders, such as drug abuse and anxiety or stress-related disorders.

It is worth mentioning that our participants appeared not to be able to accurately guess whether they had received active or sham stimulation in this single-blind tDCS design. This is noteworthy because there are currently no definite "best practices" guidelines for sham stimulation (but see Gandiga, Hummel, \& Cohen, 2006, for a study on double-blind shamcontrolled tDCS). The experience of skin sensations and side effects associated with active tDCS, as compared to sham, is thought to provide a clear clue for participants to determine whether they received active or sham stimulation. Lower impedance levels obtained during active tDCS can be expected to reduce the intensity of these skin sensations. Yet impedance levels are not typically reported in tDCS studies. In this study we were able to achieve an average impedance level of 5.15 $\mathrm{k} \Omega$, which could have aided in the inability of participants to accurately determine the stimulation condition. Unfortunately, besides asking about tolerability and assessment of the skin under the electrodes, we did not systematically administer a more comprehensive tDCS side-effects questionnaire.

Several limitations of our study design should be discussed. First, it is likely that our use of a conventional $1 \times$ 1 tDCS montage with $5 \times 5$-cm sponges modulated neighboring brain areas in addition to the OFC. For example, the 
frontopolar cortex, which based on our electrical field modeling in Fig. $1 \mathrm{~b}$ may have been stimulated, has been indicated in playing a role in counterfactual thinking (Boorman et al., 2011; Koechlin, 2014) and our findings may thus be due to stimulation over the frontal pole. Similarly, we cannot rule out that effects may have been due to anodal stimulation over EEG coordinate PO8. Future improvements in focality may be achieved using multichannel high-definition tDCS (Edwards et al., 2013).

A second issue related to tDCS as a technique is that we cannot confirm that (similar amounts of) current reached the OFC in all participants, nor the actual directionality of tDCS effects within the brain. For example, cathodal tDCS over brain regions other than the motor cortex, such as the prefrontal cortex, in order to modulate a cognitive process does not always appear to result in inhibition or a decrease of performance (Jacobson et al., 2012; Tremblay et al., 2014). Although these latter limitations are inherent to tDCS as a noninvasive brain modulation technique (Bikson et al., 2016), they warrant caution, especially for the development of individualized stimulation protocols for treatment of psychiatric phenomena (Brunoni et al., 2012; Philip et al., 2017). Our decision to use a $1 \times 1$ unilateral electrode montage (Nasseri et al., 2015) with the cathodal electrode over Fp1 and the anodal electrode placed away from the prefrontal cortex was based on prior research that aimed to target the ventromedial PFC, a region in close proximity to the OFC, and which used a close to similar placement of the electrode of interest (Hämmerer et al., 2016; van't Wout et al., 2017; van't Wout et al., 2016). We next attempted to optimize electrode placement with current density modeling prior to the study, which indicated that the currently used montage with the prefrontal electrode over Fp1 and the reference electrode over PO8 may allow current to reach the OFC. Yet future studies will need to replicate our findings and examine whether decision outcome valuation and counterfactual emotion intensity can be enhanced by anodal tDCS over the prefrontal cortex.

A third aspect worth mentioning is that, in most participants receiving active $\mathrm{tDCS}$, the 20 minutes of active stimulation (a duration chosen to stay well within safety limits of tDCS application; Bikson et al., 2016; Brunoni et al., 2011) ended before participants completed the gambling task- however tDCS effects are known to commonly outlast stimulation duration, and aftereffects have been observed up to 60 to 90 minutes after 10 minutes of active stimulation (Ardolino, Bossi, Barbieri, $\&$ Priori, 2005; Nitsche \& Paulus, 2001). In addition, aftereffects commonly correlate with the intensity and duration of stimulation (Koo et al., 2016; Monte-Silva, Kuo, Liebetanz, Paulus, \& Nitsche, 2010). Hence, we did not exclude trials on which tDCS had ceased from analyses. Although the focus of our study was not on the immediate versus aftereffects of tDCS on the decisioninduced and counterfactual affect, additional analyses on this issue are reported in the Supplementary Materials, demonstrating that $\mathrm{tDCS}$ continued to modulate outcome valuations after tDCS had ceased. An interesting but not currently examined question is whether continued engagement in the same cognitive task during and immediately after stimulation may result in more homogeneous aftereffects due to this consistency.

Future studies may want to examine if tDCS can alter the influence of anticipatory regret and relief to guide subsequent decisions (Coricelli et al., 2005), something we decided not to examine in this experiment (but see Supplementary Materials for exploratory analyses on shift/stay behavior after attained outcomes were a loss vs. win and counterfactuals resulted in regret vs. relief). In addition, it would be interesting to test whether the effect of tDCS is the same when decisionrelated (counterfactual) affect is the result of inaction instead of action (Kahneman \& Tversky, 1982; Landman, 1987; Ritov \& Baron, 1990; Spranca, Minsk, \& Baron, 1991). Finally, our results suggest that examining the effects of tDCS in individuals with aberrations in value-based decision-making is a worthwhile avenue to further test the possibilities of tDCS for future clinical applications.

Acknowledgements This work was supported in part by the Center for Neurorestoration and Neurotechnology (N9228-C) from the United States (U.S.) Department of Veterans Affairs, Rehabilitation Research and Development Service, Providence, RI. The views expressed in this article are those of the authors and do not represent the views of the U.S. Department of Veterans Affairs or the United States Government.

\section{References}

Abend, R., Jalon, I., Gurevitch, G., Sar-El, R., Shechner, T., Pine, D. S., ... Bar-Haim, Y. (2016). Modulation of fear extinction processes using transcranial electrical stimulation. Translational Psychiatry, 6(10), e913.

Ardolino, G., Bossi, B., Barbieri, S., \& Priori, A. (2005). Non-synaptic mechanisms underlie the aftereffects of cathodal transcutaneous direct current stimulation of the human brain. The Journal of Physiology, 568(2), 653-663.

Bechara, A., Damasio, H., \& Damasio, A. R. (2000). Emotion, decision making and the orbitofrontal cortex. Cerebral Cortex, 10(3), 295307.

Bell, D. E. (1982). Regret in decision making under uncertainty. Operations Research, 30(5), 961-981.

Bikson, M., Datta, A., \& Elwassif, M. (2009). Establishing safety limits for transcranial direct current stimulation. Clinical Neurophysiology: Official Journal of the International Federation of Clinical Neurophysiology, 120(6), 1033.

Bikson, M., Grossman, P., Thomas, C., Zannou, A. L., Jiang, J., Adnan, T., ... Woods, A. J. (2016). Safety of transcranial direct current stimulation: Evidence based update 2016. Brain Stimulation, 9(5), 641661.

Bogdanov, M., Ruff, C.C. \& Schwabe, L. (2015). Transcranial stimulation over the dorsolateral prefrontal cortex increases the impact of past expenses on decision-making. Cerebral Cortex. https://doi.org/ 10.1093/cercor/bhv298

Boggio, P. S., Campanhã, C., Valasek, C. A., Fecteau, S., Pascual-Leone, A., \& Fregni, F. (2010). Modulation of decision-making in a gambling task in older adults with transcranial direct current stimulation. European Journal of Neuroscience, 31(3), 593-597. 
Boorman, E. D., Behrens, T. E., \& Rushworth, M. F. (2011). Counterfactual choice and learning in a neural network centered on human lateral frontopolar cortex. PLoS biology, 9(6), e1001093.

Brunoni, A. R., Amadera, J., Berbel, B., Volz, M. S., Rizzerio, B. G., \& Fregni, F. (2011). A systematic review on reporting and assessment of adverse effects associated with transcranial direct current stimulation. International Journal of Neuropsychopharmacology, 14(8), 1133-1145.

Brunoni, A. R., Nitsche, M. A., Bolognini, N., Bikson, M., Wagner, T., Merabet, L., ... Ferrucci, R. (2012). Clinical research with transcranial direct current stimulation (tDCS): Challenges and future directions. Brain stimulation, 5(3), 175-195.

Camille, N., Coricelli, G., Sallet, J., Pradat-Diehl, P., Duhamel, J. R., \& Sirigu, A. (2004). The involvement of the orbitofrontal cortex in the experience of regret. Science, 304(5674), 1167-1170.

Cheng, G. L., \& Lee, T. M. (2016). Altering risky decision-making: Influence of impulsivity on the neuromodulation of prefrontal cortex. Social Neuroscience, 11(4), 353-364.

Chua, H. F., Gonzalez, R., Taylor, S. F., Welsh, R. C., \& Liberzon, I. (2009). Decision-related loss: Regret and disappointment. NeuroImage, 47(4), 2031-2040.

Civai, C., Miniussi, C., \& Rumiati, R. I. (2015). Medial prefrontal cortex reacts to unfairness if this damages the self: A tDCS study. Social Cognitive and Affective Neuroscience, 10(8), 1054-1060.

Clark, L., Bechara, A., Damasio, H., Aitken, M. R. F., Sahakian, B. J., \& Robbins, T. W. (2008). Differential effects of insular and ventromedial prefrontal cortex lesions on risky decision-making. Brain, 131(5), 1311-1322.

Connolly, T., Ordonez, L. D., \& Coughlan, R. (1997). Regret and responsibility in the evaluation of decision outcomes. Organizational Behavior and Human Decision Processes, 70(1), 73-85.

Coricelli, G., Critchley, H. D., Joffily, M., O’Doherty, J. P., Sirigu, A., \& Dolan, R. J. (2005). Regret and its avoidance: A neuroimaging study of choice behavior. Nature Neuroscience, 8(9), 1255-1262.

Coricelli, G., Dolan, R. J., \& Sirigu, A. (2007). Brain, emotion and decision making: The paradigmatic example of regret. Trends in Cognitive Sciences, 11(6), 258-265.

Edwards, D., Cortes, M., Datta, A., Minhas, P., Wassermann, E. M., \& Bikson, M. (2013). Physiological and modeling evidence for focal transcranial electrical brain stimulation in humans: A basis for highdefinition tDCS. NeuroImage, 74, 266-275.

Fecteau, S., Knoch, D., Fregni, F., Sultani, N., Boggio, P., \& PascualLeone, A. (2007). Diminishing risk-taking behavior by modulating activity in the prefrontal cortex: A direct current stimulation study. The Journal of Neuroscience, 27(46), 12500-12505.

Fecteau, S., Pascual-Leone, A., Zald, D. H., Liguori, P., Théoret, H., Boggio, P. S., \& Fregni, F. (2007). Activation of prefrontal cortex by transcranial direct current stimulation reduces appetite for risk during ambiguous decision making. The Journal of Neuroscience, 27(23), 6212-6218.

Fellows, L. K. (2011). Orbitofrontal contributions to value-based decision making: Evidence from humans with frontal lobe damage. Annals of the New York Academy of Sciences, 1239(1), 51-58.

Fellows, L. K., \& Farah, M. J. (2005). Different underlying impairments in decision-making following ventromedial and dorsolateral frontal lobe damage in humans. Cerebral Cortex, 15(1), 58-63.

Frank, M. J., \& Claus, E. D. (2006). Anatomy of a decision: Striatoorbitofrontal interactions in reinforcement learning, decision making, and reversal. Psychological Review, 113(2), 300-326.

Gandiga, P. C., Hummel, F. C., \& Cohen, L. G. (2006). Transcranial DC stimulation (tDCS): A tool for double-blind sham-controlled clinical studies in brain stimulation. Clinical Neurophysiology, 117(4), 845850

Gillan, C. M., Morein-Zamir, S., Kaser, M., Fineberg, N. A., Sule, A., Sahakian, B. J., ... Robbins, T. W. (2014). Counterfactual processing of economic action-outcome alternatives in obsessive-compulsive disorder: Further evidence of impaired goal-directed behavior. Biological Psychiatry, 75(8), 639-646.

Gilovich, T., \& Medvec, V. H. (1995). The experience of regret: What, when, and why. Psychological Review, 102(2), 379-395.

Hämmerer, D., Bonaiuto, J., Klein-Flügge, M., Bikson, M., \& Bestmann, S. (2016). Selective alteration of human value decisions with medial frontal tDCS is predicted by changes in attractor dynamics. Scientific Reports, 6. https://doi.org/10.1038/srep25160

Hecht, D., Walsh, V., \& Lavidor, M. (2010). Transcranial direct current stimulation facilitates decision making in a probabilistic guessing task. The Journal of Neuroscience, 30(12), 4241-4245.

Huey, E. D., Lee, S., Brickman, A. M., Manoochehri, M., Griffith, E., Devanand, D. P., ... Grafman, J. (2015). Neuropsychiatric effects of neurodegeneration of the medial versus lateral ventral prefrontal cortex in humans. Cortex, 73, 1-9.

Jacobson, L., Koslowsky, M., \& Lavidor, M. (2012). tDCS polarity effects in motor and cognitive domains: a meta-analytical review. Experimental Brain Research, 216(1), 1-10.

Kahneman, D., \& Tversky, A. (1982). The psychology of preferences. Scientific American, 246(1), 160-173.

Kempe, R., Huang, Y., \& Parra, L. C. (2014). Simulating pad-electrodes with high-definition arrays in transcranial electric stimulation. Journal of Neural Engineering, 11(2), 026003.

Koechlin, E. (2014). An evolutionary computational theory of prefrontal executive function in decisionmaking. Philosophical Transactions of the Royal Society B, 369(1655), 20130474.

Koo, H., Kim, M. S., Han, S. W., Paulus, W., Nitche, M. A., Kim, Y. H., ... Shin, Y. I. (2016). After-effects of anodal transcranial direct current stimulation on the excitability of the motor cortex in rats. Restorative Neurology and Neuroscience, 34(5), 859-868.

Landman, J. (1987). Regret and elation following action and inaction. Personality and Social Psychology Bulletin, 13(4), 524-536.

Ly, V., Bergmann, T. O., Gladwin, T. E., Volman, I., Usberti, N., Cools, R., \& Roelofs, K. (2016). Reduced affective biasing of instrumental action with tDCS over the prefrontal cortex. Brain Stimulation, 9(3), 380-387.

Mellers, B. A., \& McGraw, A. P. (2001). Anticipated emotions as guides to choice. Current Directions in Psychological Science, 10(6), 210 214.

Monte-Silva, K., Kuo, M. F., Liebetanz, D., Paulus, W., \& Nitsche, M. A. (2010). Shaping the optimal repetition interval for cathodal transcranial direct current stimulation (tDCS). Journal of Neurophysiology, 103(4), 1735-1740.

Moretto, G., Làdavas, E., Mattioli, F., \& Di Pellegrino, G. (2010). A psychophysiological investigation of moral judgment after ventromedial prefrontal damage. Journal of Cognitive Neuroscience, 22(8), 1888-1899.

Nasseri, P., Nitsche, M. A., \& Ekhtiari, H. (2015). A framework for categorizing electrode montages in transcranial direct current stimulation. Frontiers in Human Neuroscience, 9, 54.

Nitsche, M. A., Cohen, L. G., Wassermann, E. M., Priori, A., Lang, N., Antal, A., ... Pascual-Leone, A. (2008). Transcranial direct current stimulation: State of the art 2008. Brain Stimulation, 1(3), 206-223.

Nitsche, M. A., Liebetanz, D., Antal, A., Lang, N., Tergau, F., \& Paulus, W. (2003). Modulation of cortical excitability by weak direct current stimulation-Technical, safety and functional aspects. Supplements to Clinical Neurophysiology, 56, 255-276.

Nitsche, M. A., \& Paulus, W. (2000). Excitability changes induced in the human motor cortex by weak transcranial direct current stimulation. The Journal of Physiology, 527(3), 633-639.

Nitsche, M. A., \& Paulus, W. (2001). Sustained excitability elevations induced by transcranial DC motor cortex stimulation in humans. Neurology, 57(10), 1899-1901.

Philip, N. S., Nelson, B. G., Frohlich, F., Lim, K. O., Widge, A. S., \& Carpenter, L. L. (2017). Low-intensity transcranial current 
stimulation in psychiatry. American Journal of Psychiatry, 174(7), $628-639$.

Rangel, A., Camerer, C., \& Montague, P. R. (2008). A framework for studying the neurobiology of value-based decision making. Nature Reviews Neuroscience, 9(7), 545-556.

Rasmussen, S. A., \& Eisen, J. L. (1992). The epidemiology and clinical features of obsessive compulsive disorder. Psychiatric Clinics of North America, 15(4), 743-758.

Ritov, I., \& Baron, J. (1990). Reluctance to vaccinate: Omission bias and ambiguity. Journal of Behavioral Decision Making, 3(4), 263-277.

Roese, N. J., Epstude, K. A. I., Fessel, F., Morrison, M., Smallman, R., Summerville, A., ... Segerstrom, S. (2009). Repetitive regret, depression, and anxiety: Findings from a nationally representative survey. Journal of Social and Clinical Psychology, 28(6), 671-688.

Rolls, E. T., \& Grabenhorst, F. (2008). The orbitofrontal cortex and beyond: From affect to decision-making. Progress in Neurobiology, 86(3), 216-244.

Roy, M., Shohamy, D., \& Wager, T. D. (2012). Ventromedial prefrontalsubcortical systems and the generation of affective meaning. Trends in Cognitive Sciences, 16(3), 147-156.

Sachdev, P. S., \& Malhi, G. S. (2005). Obsessive-compulsive behaviour: A disorder of decision- making. Australian and New Zealand Journal of Psychiatry, 39(9), 757-763.

Simonson, I. (1992). The influence of anticipating regret and responsibility on purchase decisions. Journal of Consumer Research, 19(1), 105-118.

Spranca, M., Minsk, E., \& Baron, J. (1991). Omission and commission in judgment and choice. Journal of Experimental Social Psychology, 27(1), 76-105.

Tremblay, S., Lepage, J. F., Latulipe-Loiselle, A., Fregni, F., PascualLeone, A., \& Théoret, H. (2014). The uncertain outcome of prefrontal tDCS. Brain Stimulation, 7(6), 773-783.

Tsuchida, A., Doll, B. B., \& Fellows, L. K. (2010). Beyond reversal: A critical role for human orbitofrontal cortex in flexible learning from probabilistic feedback. Journal of Neuroscience, 30(50), 1686816875.
Ursu, S., \& Carter, C. S. (2005). Outcome representations, counterfactual comparisons and the human orbitofrontal cortex: Implications for neuroimaging studies of decision-making. Cognitive Brain Research, 23(1), 51-60.

van't Wout, M., Longo, S. M., Reddy, M. K., Philip, N. S., Bowker, M. T., \& Greenberg, B. D. (2017). Transcranial direct current stimulation may modulate extinction memory in posttraumatic stress disorder. Brain and Behavior, 7(5), e00681.

van't Wout, M., Mariano, T. Y., Garnaat, S. L., Reddy, M. K., Rasmussen, S. A., \& Greenberg, B. D. (2016). Can transcranial direct current stimulation augment extinction of conditioned fear? Brain Stimulation, 9(4), 529-536.

Volkow, N. D., \& Fowler, J. S. (2000). Addiction, a disease of compulsion and drive: Involvement of the orbitofrontal cortex. Cerebral Cortex, 10(3), 318-325.

Wallis, J. D. (2007). Orbitofrontal cortex and its contribution to decisionmaking. Annual Review of Neuroscience, 30, 31-56.

Ye, H., Huang, D., Wang, S., Zheng, H., Luo, J., \& Chen, S. (2016). Activation of the prefrontal cortex by unilateral transcranial direct current stimulation leads to an asymmetrical effect on risk preference in frames of gain and loss. Brain Research, 1648, 325-332.

Zeelenberg, M. (1999). Anticipated regret, expected feedback and behavioral decision making. Journal of Behavioral Decision Making, 12(2), 93-106.

Zeelenberg, M., Van Dijk, W. W., \& Manstead, A. S. (1998). Reconsidering the relation between regret and responsibility. Organizational Behavior and Human Decision Processes, 74(3), 254-272.

Zeelenberg, M., Van Dijk, W. W., Manstead, A. S., \& van der Pligt, J. (2000). On bad decisions and disconfirmed expectancies: The psychology of regret and disappointment. Cognition \& Emotion, 14(4), 521-541.

Zheng, H., Huang, D., Chen, S., Wang, S., Guo, W., Luo, J., ... Chen, Y. (2016). Modulating the activity of ventromedial prefrontal cortex by anodal tDCS enhances the trustee's repayment through altruism. Frontiers in Psychology, 7, 1437. 